Am. J. Trop. Med. Hyg., 73(2), 2005, pp. 423-427

Copyright $@ 2005$ by The American Society of Tropical Medicine and Hygiene

\title{
OUTBREAK OF SEVERE ACUTE RESPIRATORY SYNDROME IN SOUTHERN
}

\author{
TAIWAN, 2003
}

\author{
SHENG-NAN LU, DONALD DAH-SHYONG JIANG, JIEN-WEI LIU, MENG-CHIH LIN, CHAO-LONG CHEN, IH-JEN SU, \\ AND SHUN-SHENG CHEN* \\ Departments of Internal Medicine, Neurology, and Surgery, Kaohsiung Chang Gung Memorial Hospital, Kaohsiung, Taiwan; \\ School of Medicine, Chang Gung University, Taoyuan, Taiwan; Department of Health, Center for Disease Control, Taipei, Taiwan
}

\begin{abstract}
This study describes the epidemiologic features of the severe acute respiratory syndrome (SARS) outbreak in southern Taiwan in 2003. According to the official files of reported cases of SARS from February 21 to June 19, 2003, there were 586 cases in southern Taiwan. Symptom onset occurred between February 21 and June 19 in reported cases, between March 13 and May 30 in probable cases, and between March 17 and May 23 in polymerase chain reaction (PCR)-positive probable cases. Dates of symptom onset were earliest for six imported cases, followed by 53 cases related to nosocomial infections and 51 cases without known sources of infection. The positive rates of the PCR for these three groups decreased from $50.0 \%$ to $28.3 \%$ to $3.9 \%$, respectively $(P<0.001$, by chi-square test for linear trend). Three other cases resulted from exposure to contaminated hospitals in northern Taiwan, one of which was the index case of the nosocomial infection. Imported cases following nosocomial infection were the major cause of SARS infections in southern Taiwan. Due to the low positive rate of the PCR for SARS coronavirus, and the low positive predictive value of reported cases, the factuality of cases with unknown sources of infection should be further verified.
\end{abstract}

\section{INTRODUCTION}

Severe acute respiratory syndrome (SARS) is an emerging infectious disease that first manifested in humans in China in November $2002^{1}$ and subsequently spread worldwide. Outbreaks have occurred in China, ${ }^{1,2}$ Hong Kong, ${ }^{3,4}$ Singapore, ${ }^{5}$ Vietnam, ${ }^{6}$ Canada, ${ }^{7}$ and elsewhere for a combined total of 8,422 probable cases in 32 areas of the world, resulting in 916 confirmed deaths from this disease. In February 2003, SARS broke out in Taiwan, ${ }^{8}$ resulting in 665 cases with 180 documented deaths. ${ }^{9}$ Early in this outbreak, most cases, including some incidents of nosocomial cluster infection, ${ }^{8}$ occurred in northern Taiwan, especially in the Taipei metropolitan area. After the outbreak in northern Taiwan, the southern part of Taiwan (in the vicinity of the industrialized city of Kaohsiung) was also affected. On July 5, 2003, the World Health Organization (WHO) removed Taiwan from the list of areas with recent local transmission of SARS. The study describes the epidemiologic features of the SARS outbreak in southern Taiwan.

\section{MATERIALS AND METHODS}

Study area. Southern Taiwan includes three cities and five counties: Chiayi City and County, Tainan City and County, Kaohsiung City and County, Pingtung County, and Penghu County. Kaoshiung City is the largest metropolitan center of these areas. Penghu County is composed of the Penghu archipelago in the Taiwan straits, and is $130 \mathrm{~km}$ (a 30-minute flight) from Kaohsiung City (Figure 1).

Study subjects. On June 19, 2003, Taiwan reported its last probable SARS case to the WHO. The official records of reported SARS cases from February 21 to June 19, 2003 were obtained from the Center for Disease Control, Taiwan (TCDC). This study incorporates cases from the hospital in

\footnotetext{
* Address correspondence to Dr. Shun-Sheng Chen, Department of Neurology, Kaohsiung Chang Gung Memorial Hospital, 123 Ta-Pei Road, Niaosung 833 Kaohsiung, Taiwan. E-mail: neuron@ms2 .hinet.net
}

southern Taiwan, as well as cases from hospitals outside the study areas if the patient was a resident of southern Taiwan. A total of 694 SARS records were collected, including 60 duplicated records. After sufficient clinical observation, 48 cases were withdrawn by the reporting hospitals. Five hundred eighty-six cases were analyzed in this study. According to WHO definitions, ${ }^{10,11}$ all reported cases were reviewed by a committee for SARS diagnosis and were categorized as probable, suspected, and excluded cases.

Diagnostic criteria of suspected cases. Before May 1, 2003, any person presenting with a history of high fever $\left(>38^{\circ} \mathrm{C}\right)$ and coughing or breathing difficulty, plus a history of travel to an area with recent local SARS transmission 10 days prior to onset of symptoms, would be classified as a suspected case. ${ }^{10}$ On May 1, 2003, the WHO revised this definition to include, in addition to the previous criteria, one or more of the following exposures during the 10 days prior to onset of symptoms: 1) close contact with a person who is a suspected or probable case of SARS, 2) a history of travel to an area with recent local transmission of SARS, and 3) residing in an area with recent local transmission of SARS. ${ }^{11}$ The WHO revision also states that a person meeting the above epidemiologic criteria with an unexplained acute respiratory illness resulting in death after November 1, 2002, on whom no autopsy has been performed, would be considered as a suspected case.

Diagnostic criteria of probable cases. At first, a suspected case with radiographic evidence of infiltration consistent with pneumonia or respiratory distress syndrome (RDS) would be classified as a probable case. ${ }^{10}$ The WHO later revised the criteria as follows: 1) a suspected case of SARS with radiographic evidence of pneumonia or respiratory distress syndrome, and 2) a case had to test positive for SARS coronavirus by at least two assays. A suspected case with autopsy findings consistent with the pathology of RDS and without an identifiable cause would also be classified as a probable case. If another diagnosis could fully explain the disease, the case was then excluded.

Confirmatory tests. Throat swabs or stool samples were collected from all reported patients at the time of reporting to the government, but not every sample was adequate for a 


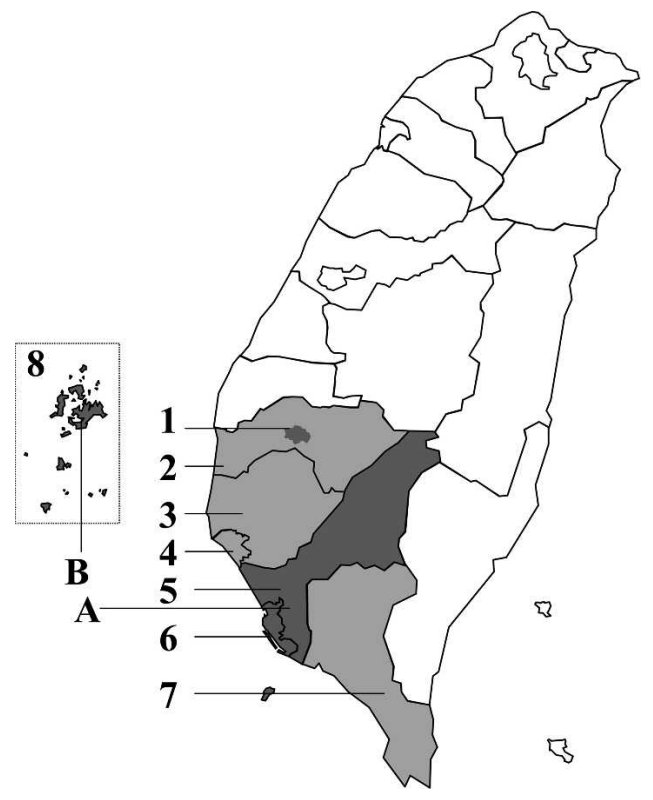

FIGURE 1. Location of study areas and hospitals in southern Taiwan. 1 = Chiayi City; 2 = Chiayi County; $3=$ Tainan County; $4=$ Tainan City; $5=$ Kaohsiung County; $6=$ Kaohsiung City; $7=$ Pingtung County; $8=$ Penghu City; $\mathrm{A}=$ Kaohsiung Memorial Hospital; $\mathrm{B}=$ Penghu Hospital. Dark gray $=$ areas with nosocomial infections; light gray $=$ areas without nosocomial infections.

polymerase chain reaction (PCR) test to detect SARS coronavirus. All probable cases with available convalescent serum were tested for antibodies to SARS coronavirus using an enzyme-linked immunosorbent assay (ELISA). However, only highly suspicious and excluded cases with atypical clinical courses were tested for confirmation. Both the PCR and antibody tests were performed by the national laboratory of the TCDC. ${ }^{12}$ Unfortunately, most of the PCR and antibody tests were not completed during the disease-endemic period, so the results of these laboratory tests were only used to corroborate the clinical diagnosis.

Associated factors of the SARS outbreak. Factors associated with the SARS outbreak included age, sex, location of reporting hospital, date of symptom onset, and associated source of infection. The sources of SARS infection were divided into four categories. All cases linked to the two hospitals with nosocomial events, either through admission, visitation, or employment, are referred to as nosocomial cases. All other cases in southern Taiwan were either imported from mainland China, Hong Kong, other countries reported to have had SARS outbreak; had a history of exposure to contaminated hospitals in northern Taiwan, or had unknown sources of infection. The positive predictive value (PPV), i.e., the percentage of probable cases of reported cases, was calculated in each group.

Nosocomial infection. In southern Taiwan, nosocomial infections were detected at two hospitals, Kaohsiung Chang Gung Memorial Hospital and Penghu Hospital. Kaohsiung Chang Gung Memorial Hospital, the largest medical center in southern Taiwan, is a 2,500-bed referral center near Kaohsiung city. Penghu Hospital is a 180-bed regional hospital located on the main island of the Penghu archipelago. The first event was induced by an unaware index case who was admitted to Kaohsiung Chang Gung Memorial Hospital on April
26, 2003 from a SARS-contaminated hospital in northern Taiwan (Jen-Chi Hospital in Taipei). The second event resulted from an asymptomatic case of lung cancer admitted to Penghu Hospital soon after being discharged from a contaminated ward of Kaohsiung Chang Gung Memorial Hospital on May 9, 2003. At Kaohsiung Chang Gung Memorial Hospital, nosocomial infections were limited to a chest ward. ${ }^{13}$ During the disease-endemic period, the following information was collected to identify the cases related to the nosocomial event: 1) complete history of all reported cases at Kaohsiung Chang Gung Memorial Hospital, 2) exposure history of all fever cases visiting the emergency services of Kaohsiung Chang Gung Memorial Hospital after the contaminated ward was closed, 3) telephone interviews with all discharged patients from the contaminated ward during the disease-endemic period, 4) computerized database from the Bureau of National Health Insurance to locate those patients discharged from Kaohsiung Chang Gung Memorial Hospital who were admitted to other hospitals in neighboring cities or counties during this period, 5) information from the mass media, and 6) official database of reported cases from the TCDC. For this investigation, we divided cases related to nosocomial infection into those with a high and low association with nosocomial infections.

Statistical analysis. Categorical data are reported as frequencies and percentages. A chi-square test was used to analyze the differences between groups and their corresponding linear trends. Dates of symptom onset were shown by epidemic curves. A type I error was set as 0.05 in all tests.

\section{RESULTS}

Of the 586 reported cases, 188 cases $(32.1 \%)$ failed to meet the WHO definition and were excluded. There were 285 (48.6\%) suspected cases, and 113 cases $(19.3 \%)$ which met the criteria of probable cases (Table 1 ). The mean \pm SD age of the patients was $50.5 \pm 22.1$ years. A total of 510 cases $(87.0 \%)$, including 97 probable cases $(85.8 \%), 231$ suspected cases $(81.1 \%)$, and 182 excluded cases $(96.8 \%)$, had adequate specimens for a reverse transcription-PCR to detect the presence or absence of SARS coronavirus. The positive rates were $21.6 \%$ ( 21 of 97 ), $3.0 \%$ ( 7 of 231 ), and $0.55 \%$ ( 1 of 182 ) in the

\section{TABLE 1}

Results of polymerase chain reaction (PCR) and antibody tests for severe acute respiratory syndrome (SARS) coronavirus by clinical diagnosis among 586 reported cases of SARS in southern Taiwan, 2003

\begin{tabular}{lrrr}
\hline & \multicolumn{3}{c}{ Antibody test result for coronavirus } \\
\cline { 2 - 4 } \multicolumn{1}{c}{ PCR for coronavirus } & Negative & Positive & None \\
\hline Probable SARS cases $(\mathrm{n}=113)$ & 57 & 5 & 14 \\
$\quad$ Negative & 11 & 7 & 3 \\
$\quad$ Positive & 5 & 1 & 10 \\
$\quad$ None & 20 & 4 & 200 \\
Suspected SARS cases $(\mathrm{n}=285)$ & 2 & 1 & 4 \\
$\quad$ Negative & 2 & 0 & 52 \\
$\quad$ Positive & & & \\
$\quad$ None & 7 & 1 & 173 \\
Excluded SARS cases $(\mathrm{n}=188)$ & 0 & 0 & 1 \\
$\quad$ Negative & 0 & 0 & 6 \\
$\quad$ Positive & & & \\
$\quad$ None & & & \\
\hline
\end{tabular}


probable, suspected, and excluded groups, respectively. Of the probable cases, $86(76.1 \%)$ were tested for antibody to SARS coronavirus and 13 of them (15.1\%) yielded positive results. Only 29 suspected $(10.2 \%)$ and 8 excluded cases $(4.3 \%)$ had atypical clinical courses and were selected for confirmation by testing for the antibody. Five suspected cases $(17.2 \%)$ and one excluded case $(12.5 \%)$ had positive results in the antibody tests. Forty showed positive results in either the PCR or antibody tests, including 27 of 113 cases probable cases $(23.9 \%), 11$ of 285 suspected cases $(3.9 \%)$, and 2 of 188 excluded cases $(1.1 \%)$.

The symptoms of the first reported case of SARS in southern Taiwan were recorded on February 21, 2003, and the last on June 19, 2003. Symptom onsets occurred between March 13 and May 30 for probable cases and between March 17 and May 23 for PCR-positive probable cases (Figure 2).

The PPV was used to evaluate the amount of disease reporting. The overall PPV was $19.3 \%$ (113 of 586). Women ( $24.1 \%$ ) 40-59 years old $(26.1 \%)$ during the period of nosocomial infection reported from areas with nosocomial infection $(25.4 \%)$ and with histories of exposure to the hospitals with nosocomial infections $(54.6 \%)$ had significantly higher PPVs. After the nosocomial infection events, only 4 (3.2\%) of 125 reported cases were diagnosed as probable cases. In analyzing the source of SARS infection, the group related to nosocomial infection in southern Taiwan had a much higher value $(54.6 \%)$ than any other group (12.0-15.8\%) (Table 2).

As shown in Table 3 , the dates of symptom onset for the six imported cases were from March 13 to May 3. Three of these cases $(50 \%)$ were PCR positive. The dates of symptom onset in the three cases exposed to a SARS-contaminated hospital in northern Taiwan were recorded on April 20, April 26, and May 24. One of them (33.3\%) was PCR positive. This was the index case of the nosocomial infection at Kaohsiung Chang Gung Memorial Hospital.

From April 26 to May 23, a total of 53 cases were defined as nosocomial infection-related cases. Fifteen $(28.3 \%)$ were confirmed as positive by PCR. Based on the epidemiologic study of the nosocomial events of Kaohsiung Chang Gung Memorial Hospital and Penghu Hospital, 35 cases had epidemiologic evidence of nosocomial events, while 8 cases had only a history of visiting the contaminated hospitals. The former 35 cases included 11 hospitalized patients, 12 family members, 3 doctors, 5 nurses, 3 nursemaids, and 1 morgue worker. The remaining 18 cases had histories of exposure to

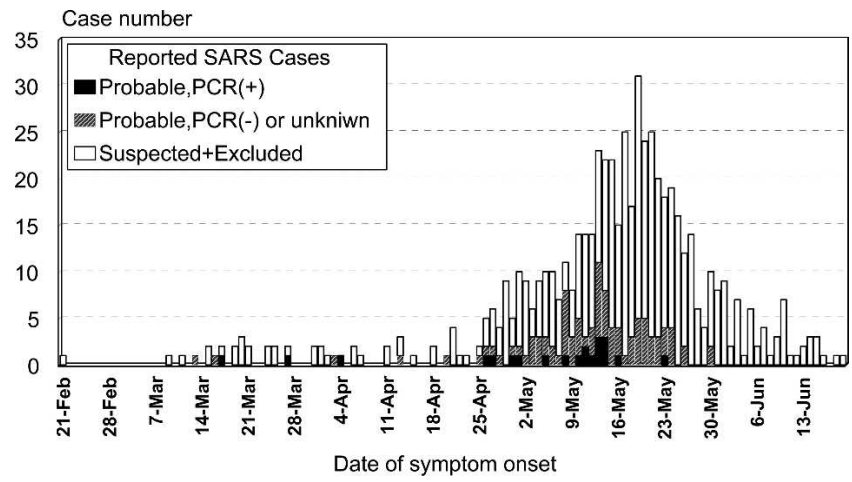

FIGURE 2. Epidemic curves of reported cases of severe acute respiratory syndrome (SARS) in southern Taiwan by grade, 2003. PCR $=$ polymerase chain reaction. either Kaohsiung Chang Gung Memorial Hospital or Penghu Hospital, but no epidemiologic association with a nosocomial infection could be established. The former 35 cases had a higher positivity rate for the PCR $(37.1 \%, 13$ of 35$)$ than the latter 18 cases $(11.1 \%, 2$ of 18$)(P=0.046)$.

The other 51 cases did not have the above-mentioned sources of infection. Their dates of onset ranged from April 3 to May 30 , and only 2 (3.9\%) of them tested positive by the PCR. As with three other cases related to SARS-contaminated hospitals in northern Taiwan, this small group was not analyzed statistically. Comparisons among the other three groups are shown in Table 3 and Figure 3. As shown in Figure 3 , imported cases were reported first, followed by cases related to nosocomial infection, and then cases without definite sources of infection. The positivity rates for the PCR for imported cases, cases related to nosocomial infection, and cases without known infection sources decreased from $50.0 \%$ to $28.3 \%$ to $3.9 \%$, respectively, $(P<0.001$, by chi-square test for linear trend).

\section{DISCUSSION}

In this study, we used the official dataset, which was reported to the WHO. The diagnoses and grading of cases were based on clinical criteria, ${ }^{10,11}$ which should be considered the major limitation of this study. The laboratory tests, including the PCR for SARS coronavirus using throat swabs and stool samples and tests for detecting antibodies to SARS coronavirus using serum samples, had not yet been adequately set up during the disease-endemic period. Well-protected laboratories for conducting the PCR for SARS were also limited, especially during the disease-endemic period. Due to the shortage of protection for SARS at the beginning of the outbreak, adequate collection of throat swabs or stool samples during the active stage of SARS was not available in a number of cases. Furthermore, the sensitivity of the PCR for SARS coronavirus was not completely satisfactory. ${ }^{14,15}$ Our PCR results were used only in correlation with clinical diagnoses in this study, since the positivity rate of the PCR for SARS coronavirus was only $21.6 \%$ in the probable cases. Although antibody test results should be positive after the window period (time between infection and the development of detectable antibody), the efficiency of immunoflorescent and enzyme immunoassays are improving. ${ }^{16,17}$ In this study, all antibody tests were conducted by the national laboratory of the TCDC, and the results have been extensively evaluated. Using a neutralization test as a reference method, we showed that the sensitivity, specificity, PPV, and negative predictive value were 98.2, 98.7, 98.7, and 98.4\%, respectively, for the ELISA. ${ }^{12}$ However, only $15.1 \%$ of the probable cases could be confirmed by the antibody test. Based on virologic or serologic tests, the number of probable cases in this study should be determined.

In our analysis, the time sequence for different causalities has been shown. Dates of symptom onset were earlier in imported cases, and later in cases related to nosocomial infection and cases with unknown sources of infection. Severe acute respiratory syndrome was first diagnosed in southern Taiwan on March 13, 2003, approximately three weeks later than in northern Taiwan. At the beginning of the outbreak, only sporadic imported cases were reported. Unfortunately, 
TABLE 2

Distributions of reported and probable cases of severe acute respiratory syndrome (SARS) and positive predictive values in southern Taiwan

\begin{tabular}{|c|c|c|c|c|c|c|}
\hline \multirow[b]{2}{*}{ Variable } & \multicolumn{2}{|c|}{ Reported case } & \multicolumn{2}{|c|}{ Probable case } & \multirow{2}{*}{$\begin{array}{c}\text { PPV* }^{*} \\
\%\end{array}$} & \multirow[b]{2}{*}{$P_{\dagger}^{\dagger}$} \\
\hline & No. & $\%$ & No. & $\%$ & & \\
\hline \multicolumn{7}{|l|}{ Sex } \\
\hline Male & 358 & 61.1 & 58 & 51.3 & 16.2 & \\
\hline Female & 228 & 38.9 & 55 & 48.7 & 24.1 & 0.018 \\
\hline \multicolumn{7}{|l|}{ Age, years } \\
\hline$\leq 19$ & 35 & 6.0 & 1 & 0.9 & 2.9 & \\
\hline $20-39$ & 173 & 29.5 & 28 & 24.8 & 16.2 & \\
\hline $40-59$ & 157 & 26.8 & 41 & 36.3 & 26.1 & 0.008 \\
\hline$\geq 60$ & 221 & 37.7 & 43 & 38.1 & 19.5 & \\
\hline \multicolumn{7}{|c|}{ Date of symptom onset: before/during/after a hospital nosocomial infection (April 27 to May 24) } \\
\hline Before & 54 & 9.2 & 11 & 9.7 & 20.4 & \\
\hline During & 407 & 69.5 & 98 & 86.7 & 24.1 & 0.001 \\
\hline After & 125 & 21.3 & 4 & 3.5 & 3.2 & \\
\hline \multicolumn{7}{|c|}{ Location of reported hospitals: areas with or without hospital nosocomial infection } \\
\hline With & 338 & 57.7 & 86 & 76.1 & 25.4 & 0.001 \\
\hline Without & 248 & 42.3 & 27 & 23.9 & 10.9 & \\
\hline \multicolumn{7}{|l|}{ Source of SARS infection } \\
\hline Imported & 44 & 7.5 & 6 & 5.3 & 13.6 & \\
\hline Nosocomial & 97 & 16.6 & 53 & 46.9 & 54.6 & 0.001 \\
\hline Unknown & 426 & 72.7 & 51 & 45.1 & 12.0 & \\
\hline Related to northern Taiwan & 19 & 3.2 & 3 & 2.7 & 15.8 & \\
\hline
\end{tabular}

$*$ PPV $=$ positive predictive value (no. of probable cases/no. of reported cases).

$\dagger$ Difference of PPV between groups; $P$ value based on chi-square test for binomial variables.

an uninformed patient from the second contaminated hospital in northern Taiwan unknowingly triggered a nosocomial infection event in the largest medical center on April 26. Although the number of reported cases increased after these nosocomial events, most lacked definite histories of exposure. Because of the efforts of the entire country, SARS was eradicated from southern Taiwan by May 30. The PCR positivity rates were subsequently determined to be $50 \%$ in imported cases ( 3 of 6 ), 33\% in cases related to northern Taiwan (1 of 3 ), and $28.3 \%$ in cases of nosocomial infection (15 of 53). However, only $3.9 \%$ of the probable patients ( 2 of 51 ) had no history of definite exposure. The PPV was quite low (3.2\%, 4 of 125) after the period of nosocomial infection. This finding points out the importance of exposure history in the diagnosis of this infectious disease.

A total of 53 cases were defined as nosocomial infectionrelated cases. These included 35 with identified epidemiologic evidence and 18 with only a history of exposure to the contaminated hospital. Our results also showed different PCR positivity rates $(37.1 \%$ versus $11.1 \%)$ between these two groups. Thus, the definite epidemiologic association was again shown to be important.
Positive predictive values were compared in this study. The last probable SARS case was reported on May 30, 2003. However, many cases continued to be reported until June 19, 2003. Before and during the period of nosocomial infection, the PPV was $>20 \%$, but it decreased to $3.5 \%$ after this period. Unfortunately, the false reports of SARS, after the reports of nosocomial infection, caused a public panic that resulted in over-reporting and over-diagnosis of the disease.

Hospital-based studies showed the transmission pattern of SARS in northern Taiwan. At first, imported cases from China transmitted the disease to family members through close household contact, and to a physician through hospital contact. ${ }^{18}$ Afterwards, nosocomial infections involved patients, accompanying family members and health care workers in the emergency service ${ }^{19}$ and wards of other involved hospitals. ${ }^{8}$ Transmission of SARS was limited when health care workers followed standard precautions or specific infection-control measures, including droplet and contact precautions. ${ }^{20}$ Effective infection-control strategies decreased nosocomial infections, and the outbreak was soon controlled. Community infection did not play an important role. ${ }^{21}$ The situation was similar in southern Taiwan. Based on clinical

TABLE 3

Dates of symptom onset and polymerase chain reaction (PCR)-positive rates among probable cases of severe acute respiratory syndrome (SARS) by their histories of exposure

\begin{tabular}{lrcrc}
\hline & \multicolumn{2}{c}{ Date of symptom onset } & \multicolumn{2}{c}{$\begin{array}{c}\text { Positive results of PCR for } \\
\text { SARS coronavirus }\end{array}$} \\
\cline { 2 - 4 } \multicolumn{1}{c}{ Source of infection } & No. & Range & No. (\%) \\
\hline Imported & 63 & March 13-May 3 & $3(50.0)^{*}$ \\
Nosocomial infections & 35 & April 27-May 24 & $15(28.3)^{*}$ \\
With high association & 18 & April 27-May 23 & $13(37.1) \dagger$ \\
$\quad$ Others & 51 & April 27-May 24 & $2(11.1) \dagger$ \\
Unknown & 3 & April 3-May 30 & $2(3.9)^{*}$ \\
Related to northern Taiwan & April 20-May 24 & $1(33.3)$ \\
\hline
\end{tabular}

* By chi-square test for a linear trend for the imported, nosocomial infections and unknown groups.

$\dagger$ By chi-square test between two groups, i.e., with or without high association with nosocomial infections. 


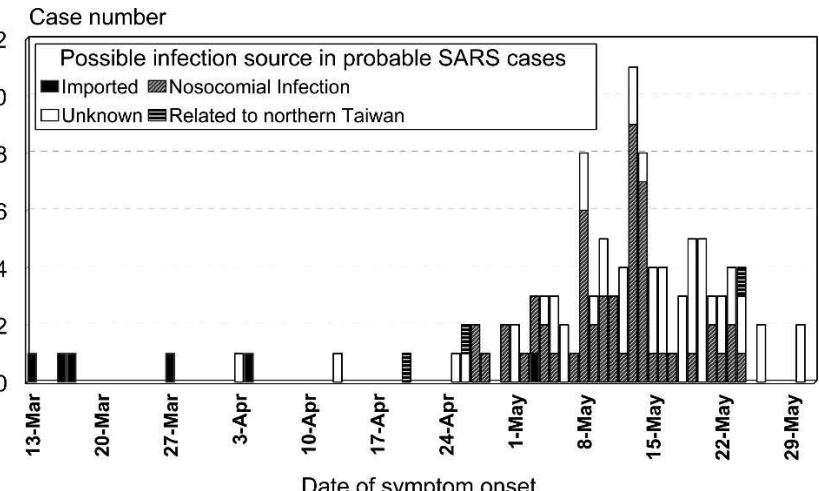

FIGURE 3. Epidemic curve of probable cases of severe acute respiratory syndrome (SARS) in southern Taiwan by possible infection sources, 2003.

diagnoses, SARS was endemic in southern Taiwan from March 13 to May 30, 2003, and the majority of cases during the initial stage were imported. Nosocomial infections and group panic related to the SARS event then followed. Due to the low positivity rates of the PCR for SARS coronavirus and low PPV, the actual number of SARS cases with unknown infection sources should be further verified.

Received November 25, 2003. Accepted for publication March 25, 2005.

Authors' addresses: Sheng-Nan Lu, Jien-Wei Liu, and Meng-Chih Lin, Department of Internal Medicine, Kaohsiung Chang Gung Memorial Hospital, Kaohsiung, Taiwan and School of Medicine, Chang Gung University, Taoyuan, Taiwan. Donald Dah-Shyong Jiang and Ih-Jen Su, Department of Health, Center for Disease Control, Taipei, Taiwan. Chao-Long Chen, Department of Surgery, Kaohsiung Chang Gung Memorial Hospital, Kaohsiung, Taiwan, and School of Medicine, Chang Gung University, Taoyuan, Taiwan. Shun-Sheng Chen, Department of Neurology, Kaohsiung Chang Gung Memorial Hospital, 123 Ta-Pei Road, Niaosung 833 Kaohsiung, Taiwan, and School of Medicine, Chang Gung University, Taoyuan, Taiwan, Telephone: 886-7-7317123 extension 3301, Fax: 886-7-7322402, E-mail: neuron@ms2.hinet.net.

\section{REFERENCES}

1. He JF, Xu RH, Yu DW, Peng GW, Liu YY, Liang WJ, Li LH, Guo RN, Fang Y, Zhang XC, Zheng HZ, Luo HM, Lin JY, 2003. Severe acute respiratory syndrome in Guangdong Province of China: epidemiology and control measures. Zhonghua Yu Fang Yi Xue Za Zhi. 37: 227-232.

2. Nie QH, Luo XD, Zhang JZ, Su Q, 2003. Current status of severe acute respiratory syndrome in China. World J Gastroenterol 9: $1635-1645$.

3. Tsang KW, Ho PL, Ooi GC, Yee WK, Wang T, Chan-Yeung M, Lam WK, Seto WH, Yam LY, Cheung TM, Wong PC, Lam B, Ip MS, Chan J, Yuen KY, Lai KN, 2003. A cluster of cases of severe acute respiratory syndrome in Hong Kong. $N$ Engl $J$ Med 348: 1977-1985.

4. Lee N, Hui D, Wu A, Chan P, Cameron P, Joynt GM, Ahuja A, Yung MY, Leung CB, To KF, Lui SF, Szeto CC, Chung S, Sung JJ, 2003. A major outbreak of severe acute respiratory syndrome in Hong Kong. N Engl J Med 348: 1986-1994.

5. Hsu LY, Lee CC, Green JA, Ang B, Paton NI, Lee L, Villacian
JS, Lim PL, Earnest A, Leo YS, 2003. Severe acute respiratory syndrome (SARS) in Singapore: clinical features of index patient and initial contacts. Emerg Infect Dis 9: 713-717.

6. Reilley B, Van Herp M, Sermand D, Dentico N, 2003. SARS and Carlo Urbani. N Engl J Med 348: 1951-1952.

7. Varia M, Wilson S, Sarwal S, McGeer A, Gournis E, Galanis E, Henry B, 2003. Hospital Outbreak Investigation Team. Investigation of a nosocomial outbreak of severe acute respiratory syndrome (SARS) in Toronto, Canada. CMAJ 169: 285-292.

8. Twu SJ, Chen TJ, Chen CJ, Olsen SJ, Lee LT, Fisk T, Hsu KH, Chang SC, Chen KT, Chiang IH, Wu YC, Wu JS, Dowell SF, 2003. Control measures for severe acute respiratory syndrome (SARS) in Taiwan. Emerg Infect Dis 9: 718-720.

9. World Health Organization, Summary table of SARS cases by country, 1 November 2002 - 7 August 2003. Available at http:// www.who.int/csr/sars/country/en/country2003_08_15.pdf. Accessed May 20, 2004.

10. WHO, 2003. Severe acute respiratory syndrome (SARS). Wkly Epidemiol Rec 78: 81-83.

11. WHO, Case Definitions for Surveillance of Severe Acute Respiratory Syndrome (SARS). Available at URL: http:// www.who.int/csr/sars/casedefinition/en/ Accessed May 20, 2004.

12. Wu HS, Chiu SC, Tseng TC, Lin SF, Lin JH, Hsu YH, Wang MC, Lin TL, Yang WZ, Ferng TL, Huang KH, Hsu LC, Lee LL, Yang JY, Chen HY, Su SP, Yang SY, Lin SY, Lin TH, Su IS, 2004. Serologic and molecular biologic methods for SARSassociated coronavirus infection, Taiwan. Emerg Infect Dis 10: 304-310.

13. Wang YH, Lin AS, Chao TY, Lu SN, Liu JW, Chen SS, Lin MC 2004. A cluster of patients with severe acute respiratory syndrome in a chest ward in southern Taiwan. Intensive Care Med. 30: 1228-1231.

14. Peiris JS, Lai ST, Poon LL, Guan Y, Yam LY, Lim W, Nicholls J, Yee WK, Yan WW, Cheung MT, Cheng VC, Chan KH, Tsang DN, Yung RW, Ng TK, Yuen KY, 2003. SARS study group. Coronavirus as a possible cause of severe acute respiratory syndrome. Lancet 361: 1319-1325.

15. Wang Y, Ma WL, Song YB, Xiao WW, Zhang B, Huang H, Wang HM, Ma XD, Zheng WL, 2003. Gene sequence analysis of SARS-associated coronavirus by nested RT-PCR. Di Yi Jun Yi Da Хие Хие Bao 23: 421-423.

16. Fang LQ, Zhang PH, Yang BA, Wu XM, Zhao QM, Liu W, Liu H, Deng YQ, Zhan L, Han WG, Lu FS, Wu JS, Yang H, Zhu QY, Cao WC, 2003. The application of indirect immunofluorescence assay in the diagnosis of severe acute respiratory syndrome. Zhonghua Liu Xing Bing Xue Za Zhi 24: 484-486.

17. Lin Y, Shen X, Yang RF, Li YX, Ji YY, He YY, Shi MD, Lu W, Shi TL, Wang J, Wang HX, Jiang HL, Shen JH, Xie YH, Wang Y, Pei G, Shen BF, Wu JR, Sun B, 2003. Identification of an epitope of SARS-coronavirus nucleocapsid protein. Cell Res 13: $141-145$

18. Hsueh PR, Chen PJ, Hsiao CH, Yeh SH, Cheng WC, Wang JL, Chiang BL, Chang SC, Chang FY, Wong WW, Kao CL, Yang PC; SARS Research Group of National Taiwan University College of Medicine and National Taiwan University Hospital, 2004. Patient data, early SARS epidemic, Taiwan. Emerg Infect Dis 10: 489-493.

19. Chen YC, Huang LM, Chan CC, Su CP, Chang SC, Chang YY, Chen ML, Hung CC, Chen WJ, Lin FY, Lee YT; SARS Research Group of National Taiwan University College of Medicine and National Taiwan University Hospital, 2004. SARS in hospital emergency room. Emerg Infect Dis 10: 782788.

20. Chen YC, Chen PJ, Chang SC, Kao CL, Wang SH, Wang LH, Yang PC; SARS Research Group of National Taiwan University College of Medicine and National Taiwan University Hospital, 2004. Infection control and SARS transmission among healthcare workers, Taiwan. Emerg Infect Dis 10: 895-898.

21. Hsueh PR, Yang PC, 2004. SARS Outbreak in Taiwan. Emerg Infect Dis 10: 1514-1515. 